\title{
A Retrospective Malaria Surveillance Data Analysis (2013- 2017), Benishangul Gumuz Region, 2018
}

\author{
Tadesse Yalew Assefa ${ }^{1,}$, , Eyoel Berhan ${ }^{2}$, Zewdu Assefa $^{1}$, Habtamu Tilahun ${ }^{1}$ \\ ${ }^{1}$ Public Health Emergency Management Center, Ethiopian Public Health Institute, Addis Ababa, Ethiopia \\ ${ }^{2}$ Department of Public Health, Saint Paul's Hospital Millennium Medical College, Addis Ababa, Ethiopia
}

Email address:

tadesseyalew021@gmail.com (T. Y. Assefa)

${ }^{*}$ Corresponding author

\section{To cite this article:}

Tadesse Yalew Assefa, Eyoel Berhan, Zewdu Assefa, Habtamu Tilahun. A Retrospective Malaria Surveillance Data Analysis (2013-2017), Benishangul Gumuz Region, 2018. Biomedical Statistics and Informatics. Vol. 5, No. 2, 2020, pp. 52-59. doi: 10.11648/j.bsi.20200502.13

Received: May 27, 2020; Accepted: June 10, 2020; Published: August 4, 2020

\begin{abstract}
Routine Malaria surveillance data is useful for assessing incidence and trends over time and in stratification for targeting of malaria. The reporting completeness and potential bias of such data needs assessment. Approximately 52 million people (68\%) live in malaria risk areas in Ethiopia, primarily at altitudes below 2,000 meters. Objective: The general objective of this analysis was to assess the trend and magnitude of Malaria surveillance data by time, place and laboratory result for the last five years in Benishangul Gumuz Region. Descriptive cross-sectional study was used to analyze Malaria surveillance data in terms of time, place and laboratory result. Data cleaning and analysis was made by using excel 2013 software. A total of 96757 expected health facilities were in the region from 2013-2017. A total of 86266 health facilities were reported in the last five years with an average completeness of $89.2 \%$. In the last five years (2013-2017) total of 1186514 clinically and confirmed malaria cases were reported regionally with 2290606 total malaria suspected fever examined with ninety inpatient deaths. Among the total clinically and confirmed cases, 1172244 (98.8\%) were outpatients, 14248 (1.2\%) were inpatients and 1013046 $(85.0 \%)$ cases were confirmed by laboratory. Out of the total confirmed malaria cases $811984(80.2 \%)$ cases were due to P.falciparum malaria and 201030 (19.8\%) cases were due to P.vivax malaria. There were no other species of malaria reported other than these two species. From the total expected health facilities; a total of 86266 health facilities were reported in the last five years with an average completeness of $89.2 \%$ which is above the WHO minimum requirement $(80.0 \%)$. The malaria reports shows that significant improvement in cases of data quality and management from year to year and currently all zones, Woredas and health facilities including private and NGO facilities are included and reported weekly the malaria surveillance from the data report identified in this study. The average five year regional incidence of confirmed malaria (P. Falciparum and P. Vivax) was less than half the incidence of the clinical and confirmed malaria cases.
\end{abstract}

Keywords: Malaria, Morbidity, Mortality, Clinical and Confirmed, Benishangul Gumuz

\section{Introduction}

Malaria is the most important of the parasitic diseases of humans, it is transmitted in 106 countries containing 3 billion people and causes approximately 2000 deaths each day; mortality rates are decreasing as a result of highly effective control programs in several countries. Malaria has been eliminated from the United States, Canada, Europe, and Russia; in the late twentieth and early twenty-first centuries, however, its prevalence rose in many parts of the tropics. Increases in the drug resistance of the parasite, the insecticide resistance of its vectors, and human travel and migration have contributed to this resurgence. Occasional local transmission after importation of malaria has occurred in several southern and eastern areas of the United States and in Europe, indicating the continual danger to nonmalarious countries. Although there are many successful new control initiatives as well as promising research initiatives, malaria remains today, as it has been for centuries, a heavy burden on tropical communities, a threat to nonendemic countries, and a 
danger to travelers [1]. A functional disease surveillance system is essential for defining problems and taking action. Proper understanding and use of this essential epidemiological tool (public health surveillance) helps health workers at the woreda and health units to set priorities, plan interventions, mobilize and allocate resources, detect epidemics early, initiate prompt response to epidemics, and evaluate and monitor health interventions. It also helps to assess long term disease trends [10].

Once malaria has been suspected or established as the cause of an epidemic or significant case buildup, notification by telephone or short-message-sending (SMS) should occur as soon as possible to all higher levels of the health system, such as the HEW supervisor, health center, district health office, zonal or regional health bureau (RHB) [11].

Routine malaria surveillance data is useful for assessing incidence and trends over time and in stratification for targeting of malaria. The reporting completeness and potential bias of such data needs assessment [2].

Stakeholders reported that malaria surveillance systems has improved in recent years, but that stronger and more reliable information systems would support elimination activities, which require intensive surveillance and tracking to promote rapid response to malaria cases [12].

Political commitments, intensified investments and control efforts in the past decade in Ethiopia have led to remarkable decline in malaria burden. The number of peripheral health facilities diagnosing and treating malaria cases, and outpatient and inpatient capacity at health centers increased dramatically. Household ownership of at least one ITN and/or households sprayed with IRS increased to $71 \%$ in 2011. In return, between 2006-2011 malaria morbidity and mortality have reduced by $>50 \%$ and $>60 \%$ respectively. The frequency and magnitude of malaria epidemics have also substantially decreased. Moreover, there has not been a malaria epidemic since 2004. The achievements made so far encouraged the government of Ethiopia to consider the inception and re-orientation of the national malaria control program towards subnational malaria eliminations with the endeavor of nationwide elimination by 2030 . To meet this goal a new malaria elimination roadmap specific to the country's context has been developed. The roadmap outlines the technical and operational measures and procedures. Confirmation of reported cases with a diagnostic test is the basis for case definition and all surveillance activities in elimination. Microscopy and RDTs are the two methods presently available for confirmatory diagnosis by the program in Ethiopia [3].

Malaria is a mosquito-borne parasitic disease that is common in the world's poorest countries. It is preventable and treatable, yet it still kills some 881,000 people every year, $90 \%$ of whom are in Africa and $85 \%$ of whom are children under five. Malaria was eliminated in most western countries more than 50 years ago; today, more than half of all estimated malaria cases occur in just five African countries: Nigeria, Democratic Republic of Congo, Ethiopia, United Republic of Tanzania and Kenya. Malaria predominantly affects rural and poor populations that have little or no access to current prevention and treatment tools. It is estimated that malaria costs Africa more than US\$12 billion every year in lost GDP, due to the heavy toll it inflicts on families in rural areas. Over the past decade or so, the world has developed several successful malaria interventions, although they remain unavailable in many places. WHO-recommended strategies for preventing and treating malaria; long-Lasting Insecticidal Nets (LLINs) are designed to repel, disable, or kill malaria-bearing mosquitoes and are effective without re-treatment for the entire life of the net (around 3-5 years), indoor Residual Spraying (IRS) employs insecticides to prevent malaria by killing mosquitoes that might bear the disease as they enter houses or other sleeping areas. Artemisinin-Combination Therapies (ACTs) are currently the most effective form of treatment against malaria.

Intermittent Preventive Treatment (IPT) in pregnancy provides antenatal malaria treatment to pregnant women, who are particularly vulnerable to the disease [4].

Approximately 52 million people (68\%) live in malaria risk areas in Ethiopia, primarily at altitudes below 2,000 meters. Malaria is mainly seasonal with unstable transmission in the highland fringe areas and of relatively longer transmission duration in lowland areas, river basins and valleys. Historically, there have been an estimated 10 million clinical malaria cases annually. Since 2006, however, cases have reduced substantially. On average, $60 \%-70 \%$ of malaria cases have been due to $P$. falciparum, with the remainder caused by $\mathrm{P}$. vivax. Anopheles arabiensis is the main malaria Vector; An. pharoensis, An. funestus and An. nili play a role as secondary vectors [5].

Ethiopia is also one of the most malaria epidemic-prone countries in Africa. Rates of morbidity and mortality increase dramatically (i.e. 3-5 fold) during epidemics. Since 2005, Ethiopia has scaled-up one of the largest and most ambitious malaria control programs in Africa, designed to support the country's Health Sector Development Plan (HSDP), the NSP and the national child survival strategy, in order to reduce under-five mortality rates by two thirds by 2015 . This SUFI phase has been possible as a result of substantial increases in resources from various funding sources and the commitment of the Government of Ethiopia (GoE). These resources have enabled an unprecedented scale-up of malaria control interventions: prompt and effective treatment, case management through rolling-out of the highly efficacious anti-malaria drugs (i.e. ACTs), and selective vector control, with a special emphasis on increasing coverage and use of ITNs, and targeted and timely application of IRS of households with insecticide [5].

Malaria transmission exhibits a seasonal and unstable pattern in Ethiopia, with transmission varying with altitude and rainfall. The major malaria transmission season in the country is from September to December, following the main rainy season from June/July to September. There is a shorter transmission season from April to May following the shorter rainy season in some parts of the country. Currently, areas 
[13].

To provide a comprehensive account of strategic information for malaria $\mathrm{M} \& \mathrm{E}$, several sources of routine data and information will be used. These sources include the following: Health Management Information System, Public Health Emergency Management/ Surveillance: Malaria M\&E is also being facilitated through the Public Health Emergency Management system (PHEM), which encompasses reporting of morbidity and mortality data from health posts, health centers and hospitals. It is a unique reporting system intended to improve the surveillance and control of 18 diseases which are either epidemic prone, targeted for eradication or of public health importance, including malaria which is one of the weekly reportable diseases. There is a distinction between diseases which are immediately reportable (e.g. polio, measles); those which are reportable weekly and those reportable monthly. Malaria indicators comprise a significant proportion of the PHEM indicators. Malaria cases are reported including clinical malaria (outpatient and inpatient), confirmed malaria by species and severe malaria. The advantage of this system is that it is a simple direct channel reported weekly. Responsibility for the PHEM system is transferred from the FMOH IDSR to the Ethiopian Public Health Institute (EPHI) PHEM. There are a clear guidelines and specific targets for timeliness and completeness of reporting, Integrated Pharmaceuticals Logistical System, Activity and Performance Reports, Sentinel Site Surveillance and Community Health Information System [6].

Several levels of data are being collected using standardized data collection tools as part of the malaria program M\&E system: household, community, health facility, and special studies. Data are entered into computer, cleaned and analyzed. Then information are being stored safely and/ or shared with appropriate levels. Information collected through HMIS is organized under the following data flow structure for all health related data according to the national HMIS guidelines: health posts and health centers (PHCU) report to the woreda. Woreda-based hospitals report to the woreda in which they are located. Other hospitals report to the zone or to the region. Woredas report to the zone or the region and Zones report to regions. Regions report to the FMOH [6].

The burden of malaria has been increasing due to a combination of large population movements, increasing large-scale epidemics, mixed infections of Plasmodium vivax and P. falciparum, increasing parasite resistance to malaria drugs, vector resistance to insecticides, low coverage of malaria prevention services, and general poverty. Outpatient consultations, inpatient admissions and all in-patient deaths have risen by $21-23 \%$ over the last five years [7]. Besides, information, and utilization of analyzed data regarding malaria is not sufficient. Therefore, it is essential to carry out this study to describe magnitude of the disease in the region and provide a plat form for policy makers for necessary action to be taken.

\section{Method}

\subsection{Study Area and Population}

The analysis was made in Benishangul gumuz region which is located in the north western part of Ethiopia and is bordered by Amhara region to the north and the northeast, Oromia region to the south and southeast and Sudan to the west. The region is comprised of three administrative zones and one special woreda made up of 21 districts and one city administration. The regional capital, Assosa is about $676 \mathrm{~km}$ west of Addis Ababa. The total population of the region is 784,345 from 2007 census. As based on 2007 census, 2017 projection is about $1,066,001$. The population of the region consists of diverse ethnic groups, five of which are indigenous to the region namely; Berta, Gumuz, Shinasha, Mao and Komo. Most of the region is situated $580 \mathrm{~m}-2730 \mathrm{~m}$ above sea level and endowed with huge tracts of forest, agricultural land and water. The region is also known for its rich gold and marble resources. The grand Ethiopian renaissance dam project is also situated in the region.

Benishangul Gumuz has an estimated area of 51,000 square kilometers. The state has diverse topography and climate. That includes the familiar traditional zones - "kola", "dega", and "woyna dega". "About $75 \%$ of the State is classified as "kola" (law lands) which is below 1500 meters above sea level. The altitude ranges from 550 to 2,500 meters above sea level. The average annual temperature reaches from $20-25^{\circ} \mathrm{C}$. During the hottest months (January - May) it reaches a $28-34^{\circ} \mathrm{C}$. The pick malaria transmission seasons are October to December followed by March to April. Both P. Vivax and P. Falciparum exist in the region.

\subsection{Study Period}

Five year (2013-2017) Malaria surveillance data collected from Benishangul Gumuz region public health emergency management was analyzed and interpreted from 30 July to 20 August 2018.

\subsection{Study Design}

Descriptive cross-sectional study was used to analyze the surveillance data in terms of time, place and laboratory result.

\subsection{Data Source}

Five year secondary data were obtained from EPHI (PHEM) surveillance data of 2013-2017. Variables such as zones, districts, clinical and confirmed, inpatient, outpatient, $P$. Falciparum and P. Vivax, malaria suspected cases and malaria deaths are included in the data base.

\subsection{Data Analysis Procedure}

Data was obtained from EPHI public health emergency data unit. The data was cleaned and entered to office excel (Laboratory result and total number of cases entered in an electronic data base) reported to Public Health Emergency Management center (PHEM) in EPHI from the year 2013- 
2017 malaria surveillance data was used for analysis. Data cleaning and analysis was made by excel 2013 software.

\subsection{Operational Definitions}

Case definition

Confirmed malaria case: A suspected case confirmed by either RDT/microscopy for isolation of plasmodium parasites.

Malaria suspected case: A person with a fever or fever with headache, chills, rigor, back pain, sweats, myalgia, nausea and vomiting diagnosed clinically as malaria.

Clinically and confirmed case: Malaria suspected cases plus confirmed malaria cases.

Malaria recurrence: Person who had exposed two or more times either by Pf. or Pv. in the same year

Minimum completeness requirement: according to WHO among expected health facilities if $>=80 \%$ of health facilities of reported.

\subsection{Ethical Consideration}

Permission approval of the study analysis was obtained from EPHI public health emergency management center.

\section{Result}

\subsection{Report Completeness of the Data}

The report completeness rate was better in 2017 reporting year in the regionally. A total of 96757 expected health facilities were in the region from 2013-2017. From the total expected; a total of 86266 health facilities were reported in the last five years with an average completeness of $89.2 \%$ which is above the WHO minimum requirement $(80.0 \%)$.

The year 2013 had reported health facilities without expected health facilities from week 25-27 as shown below figure one which may be data entry error (it was recorded as zero data). Reporting year of 2015 had no reports at all from week 14 and 16 and from week 19-21. The highest report completeness was reported in 2017 WHO reporting week of forty seven $(98.8 \%)$.

Assosa zone had the highest average zonal completeness, $94.8 \%$ followed by Maokomo special woreda (90.6\%) in the five year reporting period.

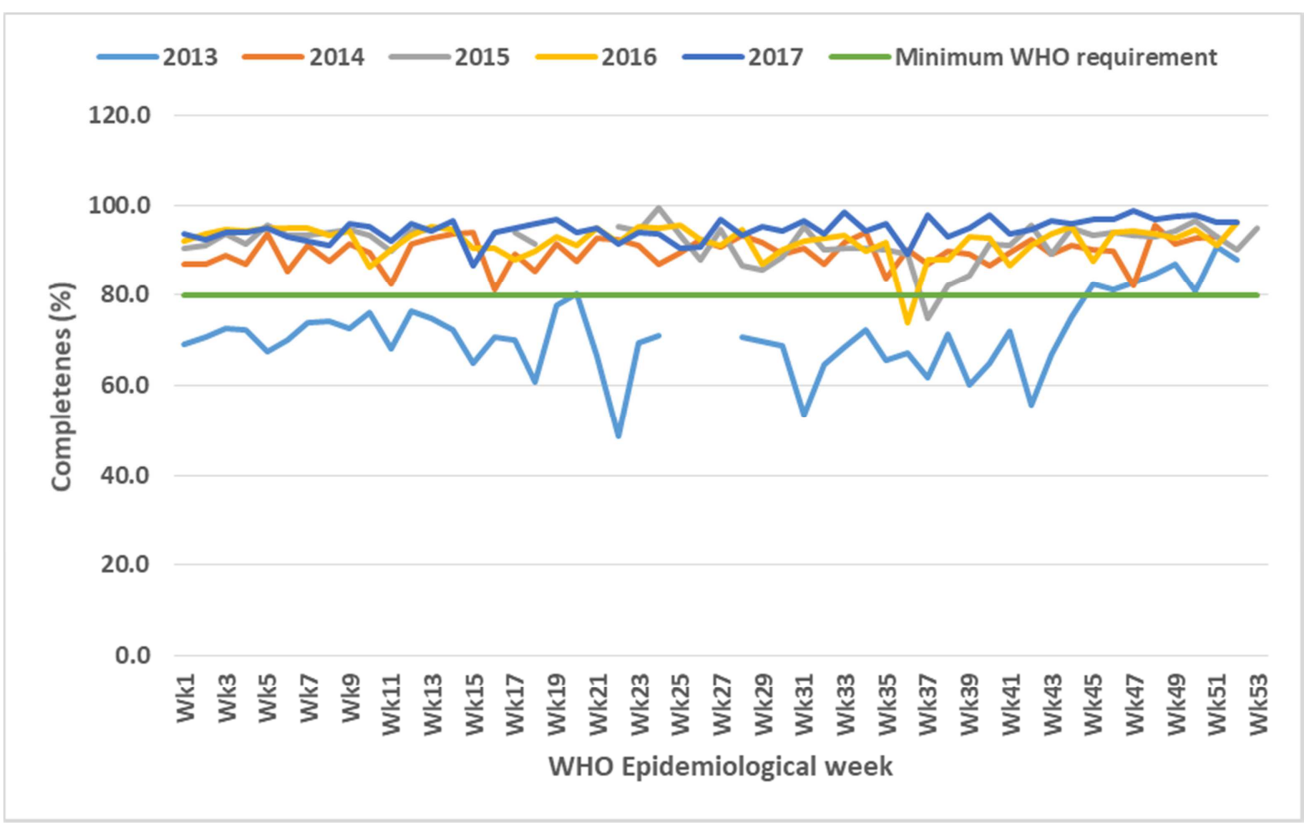

Figure 1. Report completeness by week and year, Benishangul gumuz Region, from week 1-53, 2013-2017.

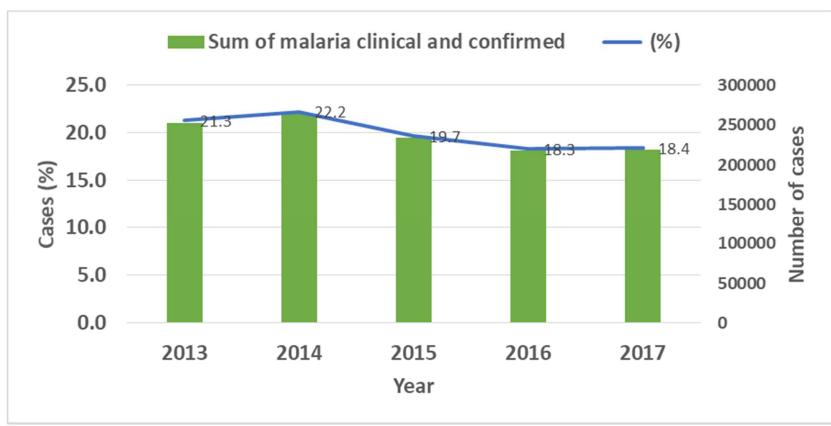

Figure 2. Total Malaria cases, clinical and confirmed, by year, Benishangul Gumuz region, from 2013-2017 GC.

\subsection{Malaria Morbidity}

In the last five years (2013-2017) total of 1186514 clinically and confirmed malaria cases were reported regionally with 2290606 total malaria suspected fever examined with ninety inpatient deaths. Among the total clinically and confirmed cases, 1172244 (98.8\%) were outpatients, $14248(1.2 \%)$ were inpatients and 1013046 $(85.0 \%)$ cases were confirmed by laboratory.

Regionally, there was a substantial increase in the number of cases from 2013-2015 (figure 3). The year with highest number of cases reported was 2014 with a total of 263977 $(22.2 \%)$ with the highest cases reported in week forty eight $8680(3.3 \%)$ clinical and confirmed cases. The year with no 
reports was 2015 with weeks of 14, 16 and 19-21 epidemic weeks. The analyzed data shows $1149(0.1 \%)$ increase in the clinically and confirmed cases in 2017 compared to 2016.

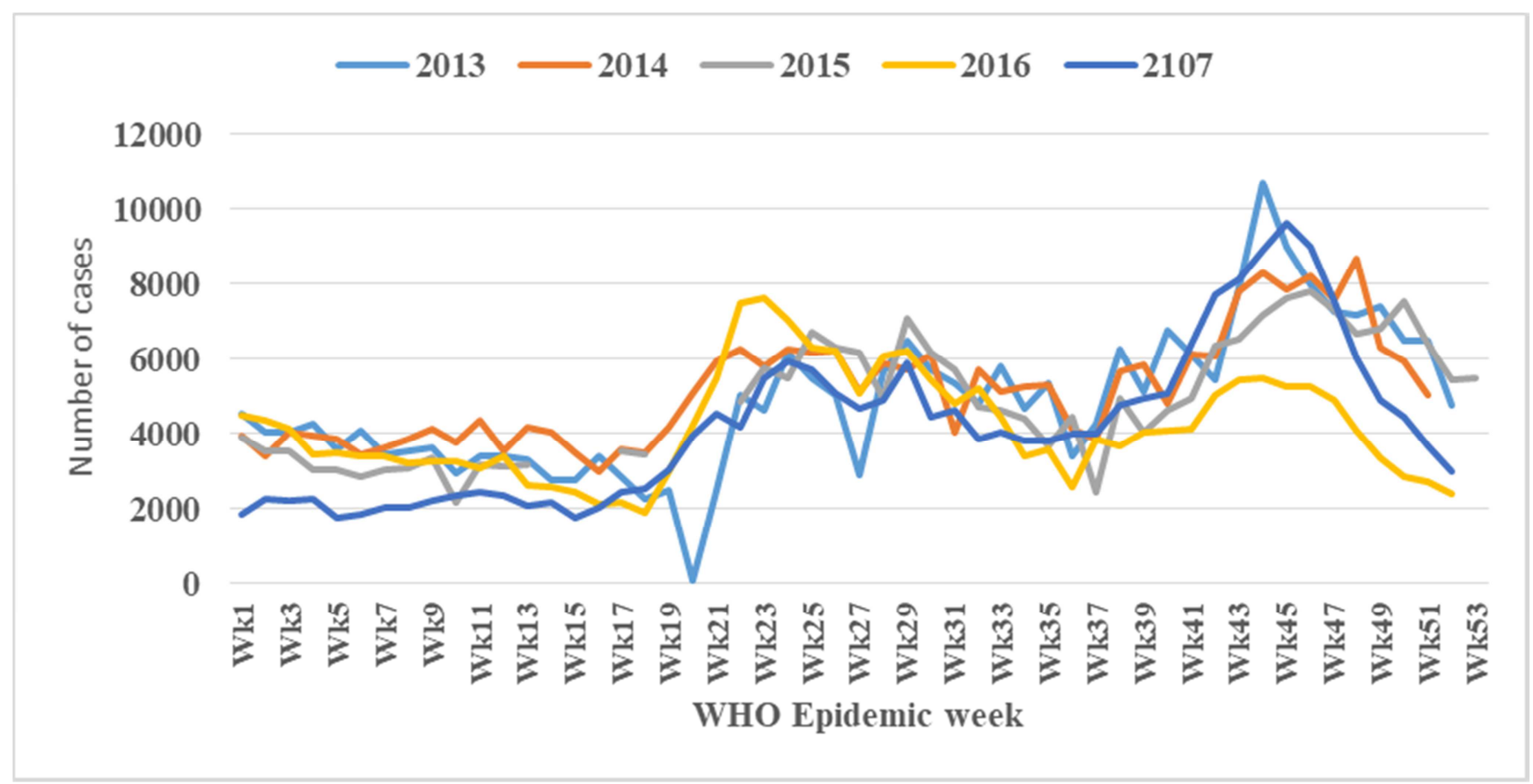

Figure 3. Trend of Malaria cases (clinical and confirmed), Benishangul gumuz Region, from week 1-53 and Year, 2013-2017.

The overall five year average of confirmed and clinically reported malaria incidence was 237.01 cases per 1000 population, which was almost parallel to the out-patients reported cases (234.13 cases per 1000 population). The trends of confirmed and clinically malaria cases incidence increase in the first two years, then declining from 232.66 cases per 1000 population in 2015 to 204.98 cases per 1000 population in 2017 (Table 1). The total malaria inpatient cases and total malaria deaths accounts 2.88 per 1000 and 0.18 per 10,000 respectively. The total malaria positive report shows an average of 201.45 per 1000; with an average of P.falciparum 161.09/1000 and P.vivax 40.37 cases per 1000 respectively.

Table 1. Total five year Malaria incidence by year, Benishangul Gumuz Region, 2013-2017.

\begin{tabular}{|c|c|c|c|c|c|c|}
\hline \multicolumn{7}{|c|}{ Total Malaria cases and Incidence by Indicators Benishangul Gumuz Region, 2013-2017 } \\
\hline \multirow{2}{*}{ Indicator } & \multicolumn{5}{|c|}{ Year } & \multirow{2}{*}{$\begin{array}{l}\text { Average } \\
\text { incidence }\end{array}$} \\
\hline & 2013 & 2014 & 2015 & 2016 & 2017 & \\
\hline Total confirmed and clinical Malaria cases $/ 1000$ & 266.76 & 270.47 & 232.66 & 210.21 & 204.98 & 237.01 \\
\hline Total outpatient Malaria cases $/ 1000$ & 262.14 & 267.07 & 230.32 & 207.51 & 203.63 & 234.13 \\
\hline Total inpatient Malaria cases/1000 & 4.62 & 3.40 & 2.34 & 2.70 & 1.32 & 2.88 \\
\hline Total Malaria inpatient deaths $/ 100000$ & 0.24 & 0.20 & 0.28 & 0.11 & 0.80 & 0.18 \\
\hline Total Malaria Positive cases/1000 & 191.88 & 211.03 & 210.79 & 193.45 & 200.13 & 201.45 \\
\hline Total P.falciparum cases $/ 1000$ & 141.43 & 159.04 & 171.12 & 161.06 & 172.79 & 161.09 \\
\hline Total P.vivax cases $/ 1000$ & 50.45 & 51.98 & 39.68 & 32.38 & 27.34 & 40.37 \\
\hline Total Malaria suspected febrile cases examined/1000 & 334.85 & 418.07 & 448.12 & 489.80 & 570.7 & 452.31 \\
\hline
\end{tabular}

\subsection{Malaria Mortality}

Regionally, from 2013-2017 there were a total of 90 inpatient malaria deaths reported. The mean number of malaria deaths per year was 18 . The Regional malaria deaths range between $31.1 \%$ in 2015 with WHO epidemic week of thirty two followed by 2013 (25.6\%) in WHO epidemic week of week forty two (figure 4). The zonal distribution of deaths was highest in Metekel zone (56.67\%) followed by Assosa zone $(30.0 \%)$ (figure 5) and no deaths were reported in Maokomo special woreda. The fatality rate was higher in Metekel zone (10.1 per 100000) followed by Assosa zone (6.1 per 100000) and the least in Maokomo special woreda (Table 2). 


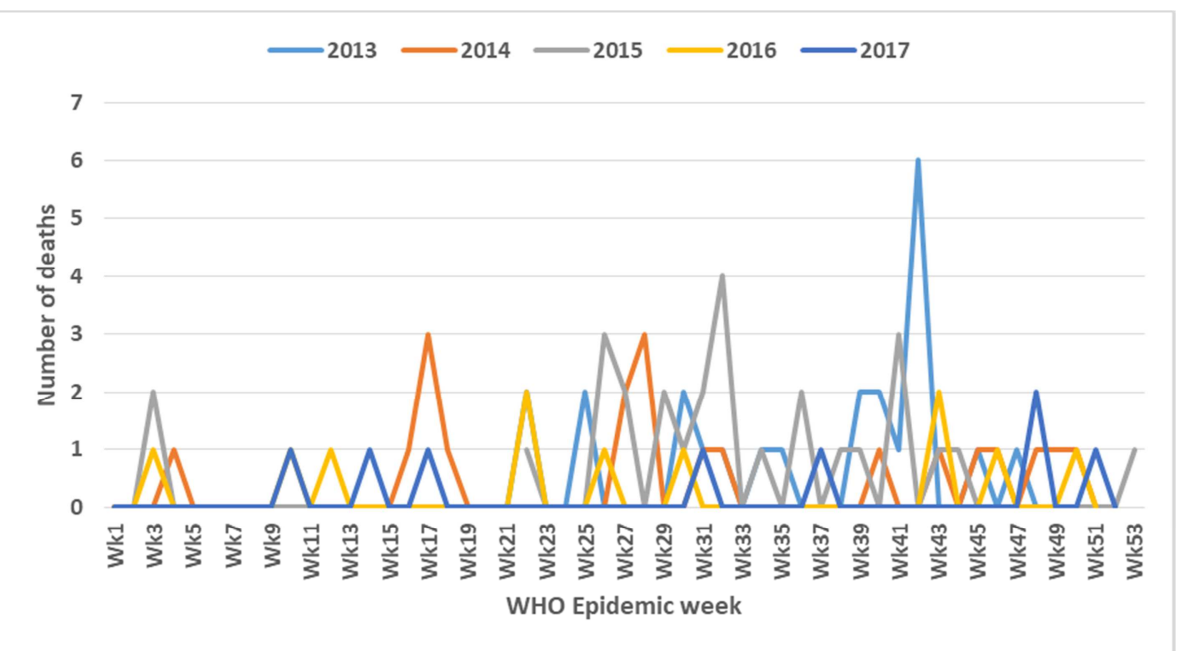

Figure 4. Trend of Malaria cases (clinical and confirmed), Benishangul Gumuz Region, from week 1-53 and Year, 2013-2017.

Table 2. Malaria death by zone with fatality rate, Benishangul Gumuz region, 2013-2017.

\begin{tabular}{llllllll}
\hline Zone name & Year & & & & Total & Fatality rate/100000 \\
\hline & $\mathbf{2 0 1 3}$ & $\mathbf{2 0 1 4}$ & $\mathbf{2 0 1 5}$ & $\mathbf{2 0 1 6}$ & $\mathbf{2 0 1 7}$ & & \\
\hline Assosa & 11 & 5 & 0 & 7 & 14 & 27 & 6.1 \\
Kamashi & 4 & 2 & 2 & 1 & 3 & 12 & 6.0 \\
Maokomo Speci. & 0 & 0 & 0 & 0 & 0 & 0 & 0.0 \\
Metekel & 8 & 13 & 26 & 3 & 1 & 51 & 10.1 \\
Grand total & 23 & 20 & 28 & 11 & 18 & 90 & 7.59 \\
\hline
\end{tabular}

October and July had the highest report of deaths with a total of $18(20.0 \%)$ and $16(17.78 \%)$ deaths respectively in the five year reporting period. The month with no deaths reported was February. Malaria deaths among Woredas was much different in magnitude and only fifteen Woredas had reported malaria deaths from thirty six reporting sites; the highest malaria deaths reported were in Pawe hospital 24 (26.7\%) followed by Dangur woreda 17 (18.9\%) respectively.

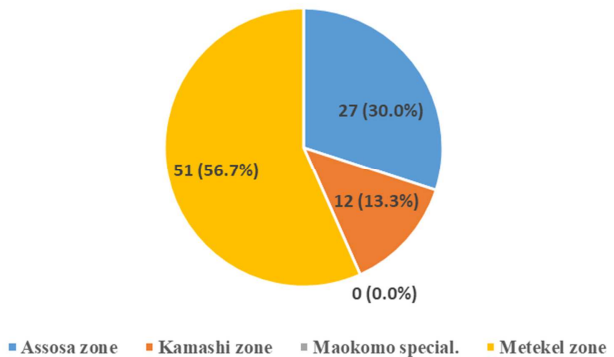

Figure 5. Total five year Malaria deaths by Zone, Benishangul Gumuz Region, 2013-2017].

\section{Discussion}

This data analysis examined the completeness of reporting of malaria indicators for the integrated diseases surveillance in Benishangul gumuz region between 2013 and 2017. From the analysis it concluded that over $80 \%$ (WHO minimum requirement), which was sufficient for quality to provide estimates of malaria incidence by reporting unit (usually by zone) and weekly to the end of 2017. The year which achieved below the WHO requirement in most of the epidemic week was 2013 except epidemic weeks of week 20 and from week 45-52, which achieved the WHO minimum requirements.

The results shows marked decline in numbers of malaria and malaria related inpatients and deaths over the period (2015-2016) except in slight increase in the number of cases in $2017,1149(0.1 \%)$ when compared to 2016 and with the highest cases recorded in 2014 which may be due to the improvement in the reporting system, perhaps as a result of scaled up interventions in the previous years; a survey conducted in Ethiopia and Uganda shows The malaria burden has declined in the past decade. Malaria mortality rates decreased by $47 \%$ globally and by $54 \%$ in the WHO African Region between 2000 and 2013 that factors that are believed to have had a significant on this trend impact include the scale-up of key vector control interventions, availability of rapid diagnostic tests (RDTs) and effective treatment with artemisinin-based combination therapy (ACT). However, the trend has not been uniform. In some areas with high baseline transmission and/or where high coverage levels have not been achieved, the malaria burden has not declined [8]. The Meher season in Ethiopian (September to November) have the most transmission of malaria followed by March to May, Ethiopian Belg season. Generally, October was the peak of the month with the highest malaria report followed by November from the total five year clinical and confirmed malaria reports which is similar with the peak of malaria incidence follows the main rainfall season (July - September) each year. However, many areas in the south and west of the country have a rainfall season beginning earlier in April and 
May or have no clearly defined rainfall season [9].

Regionally, the burden of malaria was highly concentrated in Metekel and Assosa zones and Dangur and Pawe Woredas which accounts $(42.7 \%),(37.3 \%)$ and $(9.3 \%)$ and $(8.1 \%)$ respectively.

Out of the total confirmed malaria cases $(80.2 \%)$ were due to P.falciparum malaria and $201030(19.8 \%)$ cases were due to P.vivax malaria. There were no other species of malaria reported other than these two species. P.falciparum was highest in all zones and the highest number of P.falciparum was reported in Metekel zone (45.5\%). The highest number of P.vivax was reported from Assosa zone from all zones $105178(52.3 \%)$. Excellent surveillance and response are the keys to achieving and maintaining malaria elimination; information systems must become increasingly "granular" to allow identification, tracking, classification and response for all malaria cases [14].

The list of diseases and syndromes in the national health information system (HIS) is useful for planning and routine management but too extensive for effective and useful surveillance in view of the limited human and financial resources. Therefore, depending on the objectives of the system, priority diseases for surveillance should be identified and reviewed regularly to ensure they remain relevant and important [15].

The limitation of the data analysis includes; the data had no important variables like age and sex, some weeks had no reports which may affects the completeness and representativeness of the data, the expected and the reported health facilities overlap in some weeks and years of reporting sites (usually zones). The reporting system does not specify how much of the cases are confirmed by either RDT or microscopy rather it is a combination of both diagnostic tests.

\section{Conclusion}

The report completeness rate was better in 2017 reporting year in the region. A total of 96757 expected health facilities were in the region in the total five years. From the total expected health facilities; a total of 86266 health facilities were reported in the last five years with an average completeness of $89.2 \%$ which is above the WHO minimum requirement $(80.0 \%)$.

The malaria reports shows that significant improvement in cases of data quality and management from year to year and currently all zones, Woredas and health facilities including private and NGO facilities are included and reported weekly the malaria surveillance from the data report identified in this study. Generally the strategy for control and preventive of malaria, malaria elimination surveillance and operational plan in Ethiopia had been on the right track that will and results significant decline of morbidity and mortality, including decreasing the burden of malaria cases in health facilities. The trends of confirmed and clinically malaria cases incidence increase in the first two year. Metekel zone was the only zone having the highest of malaria cases in the total five years followed by Assosa zone. From the total 35 reported Districts Dangur woreda had the highest report of clinical and confirmed malaria followed by Pawe woreda. The Meher season in Ethiopian (September to November) have the most transmission of malaria in the region followed by March to May, Ethiopian Belg season.

The average five year regional incidence of confirmed malaria (P. Falciparum and P. Vivax) was less than half the incidence of the clinical and confirmed malaria cases. The highest recorded incidence rate of confirmed malaria cases was in 2014 and 2015 years of reporting. The incidence of inpatient malaria cases had been declining since 2014. Regionally, there was a substantial increase in the number of cases from 2013-2015. The highest number of cases reported was 2014 with the highest cases reported in week forty eight clinical and confirmed cases. The year with no reports was 2015 with weeks of 14, 16 and 19-21 epidemic weeks. The analyzed data shows some cases increment in the clinically and confirmed cases in 2017 compared to 2016. The incidence of confirmed and clinical malaria cases was highest in Kamashi zone followed by Metekel zone both clinical and confirmed. The mean number of malaria deaths per year was 18. The zonal distribution of deaths was highest in Metekel zone followed by Assosa zone and no deaths were reported in Maokomo special woreda. The fatality rate was higher in Metekel zone followed by Assosa zone and the least in Maokomo special woreda.

\section{Abbreviations}

ACTs Artemisinin-Combination Therapies

BGRHB Benishangul Gumuz Regional Health bureau

EFETP Ethiopian field epidemiology training programme

EPHI Ethiopian public health institute

GC Gregorian calendar

GDP Gross domestic product

HMIS Health information management system

HSDP Health sector development plan

IDSR Integrated diseases surveillance and response

IPT Intermittent preventive treatment

IRS Insecticide residual spray

LLINs Long-Lasting Insecticidal bed Nets

$\mathrm{MOH} \quad$ Ministry of health

NGOs Non-governmental organizations

PF Plasmodium falciparum

PHCU Primary health care unit

PHEM Public health emergency management

PV Plasmodium vivax

RDT Rapid diagnostic tests

WHO World health organization

\section{Conflict of Interest}

The authors declare that there is no competing interests.

\section{Acknowledgements}

We would like to thank Ethiopian public health institute 
PHEM department for providing the weekly data for the analysis and BGRHB PHEM case team for providing important information's additionally.

\section{References}

[1] R. Harrison T, S Fauci A, L Kasper D, L Longo D. Harrison's Principles of Internal Medicine. Harrison's Principles of Internal Medicine. 2012.

[2] Jima D, Wondabeku M, Alemu A, Teferra A, Awel N, Deressa $\mathrm{W}$, et al. Analysis of malaria surveillance data in Ethiopia: What can be learned from the Integrated Disease Surveillance and Response System? Malar J [Internet]. 2012; 11 (1): 1. Available from: Malaria Journal.

[3] Elimination_Surveillance_Manual.

[4] World Malaria Report 2008. Malaria: a Global Burden. 2008; $1-2$.

[5] Edition T, Ababa A. Third Edition. Natl Malar Guid lines [Internet]. 2012; third edit (January): 1-104. Available from: http://www.qiagen.com/products/catalog/sampletechnologies/protein-sample-technologies/detection-kits-andantibodies/penta-his-antibody-bsa-free.

[6] Ababa A. National Malaria Control Program Monitoring and Evaluation Plan 2008-2013. Draft v14 [Internet]. 2009; (June): 1-43. Available from: papers2://publication/uuid/5F7F5ACDD77C-4B46-AE26-40712DCC111C.
[7] PMI. President' S Malaria Initiative Technical. Cdc. 2016; 9 11.

[8] Abeku TA, Helinski MEH, Kirby MJ, Kefyalew T, Awano T, Batisso E, et al. Monitoring changes in malaria epidemiology and effectiveness of interventions in Ethiopia and Uganda: Beyond Garki Project baseline survey. Vol. 14, Malaria Journal. 2015.

[9] Jima D, Getachew A, Bilak H, Stekeete RW, Emerson PM, Graves PM, et al. Malaria indicator survey 2007, Ethiopia: coverage and use of major malaria prevention and control interventions. Vol. 9, Malar J. 2010. p. 58.

[10] Public Health Emergency Management. 2012.

[11] Disease Surveillance. Disease Surveillance. 2016.

[12] PATH MACEPA. Ethiopia: Accelerating Toward Malaria Elimination Stakeholder Perspectives. 2015; Available from: http://www.makingmalariahistory.org/wpcontent/uploads/2016/03/Ethiopia-Stakeholder-AnalysisEXTERNAL-FINAL.pdf.

[13] Ababa A. NATIONAL STRATEGIC PLAN FOR MALARIA PREVENTION. 2015; 2015 (August 2010).

[14] World Health Organization, Global Malaria Programme. A Framework for Malaria Elimination [Internet]. Geneva World Health Organization. 2017. 100 p. Available from: http://apps.who.int/iris/bitstream/handle/10665/254761/97892 41511988-eng.pdf?sequence $=1$.

[15] Cummings M. Communicable Disease Surveillance and Response in Uganda. Cons J Sustain Dev. 2009; Issue (Two). 